Sie haben Ihre Anwaltszulassung längere Zeit ruhen lassen, praktizieren mittlerweile aber wieder. Wie gestaltet sich Ihr Berufsalltag heute?

Ich habe meine Zulassung seit September 2007 wieder. Aber ich habe keine Kanzlei, kein Büro, wie ich es bisher hatte. Nach wie vor habe ich Angst, im selben Stil zu arbeiten wie bis zur Schließung der Kanzlei. Mein Berufsalltag hat sich verschoben. Ich konzentriere mich auf das Schreiben und schreibe wieder ein neues Buch. Nebenbei berate ich Frauen, die in einer extremen Notsituation sind. Es melden sich bei mir ausschließlich Frauen, die von Zwangsverheiratung, Ehrenmord und häuslicher Gewalt betroffen sind, und zwar bundesweit. Entweder bekomme ich direkt von den Frauen eine Mail oder Ärzte(innen), Psychologen(innen), Sozialarbeiter(innen), Lehrer(innen) etc. melden sich zunächst und ich nehme dann Kontakt mit den Frauen auf, wenn sie das wünschen. Meinen Lebensunterhalt verdiene ich sozusagen inzwischen als Autorin.

Haben Sie Anregungen für andere Frauen in ähnlicher Situation, für die die beruflichen Umstände (fast) untragbar geworden sind, aber die wie Sie nicht aufgeben wollen?
Sie können sich nur um den größtmöglichen Schutz kümmern, den sie selbst organisieren können und sich hin und wieder mit anderen austauschen. Etwas anderes sehe ich nicht.

\section{Gibt es einen Wunsch, den Sie an den djb haben?}

Es wäre schön, wenn der djb das Thema Gewalt an Kolleginnen immer wieder aufgreifen würde und Kolleginnen ansprechen, ermutigen würde, ihre Fälle dem djb mitzuteilen. Nur wenn wir davon wissen, können wir sagen, dass es nach wie vor betroffene Kolleginnen gibt und Handlungsbedarf besteht. Auch die latente Angst, unter der einige Kolleginnen arbeiten, weil sie sich mit prekären Themen beschäftigen, ist eine Form von Gewalt.

Liebe Frau Ateş, ich bin mir sicher, vor allem Jutta Wagner, aber auch wir anderen alle werden unser Bestes tun! Ihnen weiterhin viel Kraft bei der Umsetzung all der Dinge, die sie sich vorgenommen haben. Der Tatort „Familienaufstellung“, der unter Ihrer Mitwirkung entstand und am Sonntag, den 8. Februar 2009 in der ARD gezeigt wurde, ist sicherlich nicht nur vielen von uns djb-Mitgliedern in Erinnerung geblieben. Vielen Dank für das Interview.

\title{
Binationale Ehen in der Beratungspraxis
}

\section{- der Ehegattennachzug zu einem deutschen Ehepartner}

In die anwaltliche Beratungspraxis kommen die deutsch-deutschen Ehepaare selten mit dem romantisch-verklärten Ziel, sich beraten zu lassen, weil sie verliebt, verlobt, verheiratet sind und sich lieben wollen, bis der Tod sie scheidet. Vielmehr kommt ein Teil des Paares, weil die Ehe zerrüttet ist oder sie/er die Ehe für zerrüttet hält oder ihr/ihm bereits durch ein Anwaltsschreiben die Zerrüttung der Ehe dokumentiert wurde. Die Beispiele lassen sich beliebig fortsetzen. Bei allen diesen Paaren war aber die Eheschließung, sei es eine Zweckehe wegen Ehegattensplittings oder eine Versorgungsehe wegen des Rentenanspruchs, eine freie Entscheidung der gemeinsamen Lebensform, während es für die - auch in diesem Beitrag ausschließlich fokussierten - Ehen zwischen einem deutschen und einem nichteuropäischen Staatsbürger charakteristisch ist, dass dieses Paar keine Möglichkeit hat, sich zu entscheiden, in welcher Form es ihr gemeinsames Leben führen möchte. Denn wenn sie zusammen leben möchten, müssen sie meist heiraten. Das Aufenthaltsgesetz sieht keine Möglichkeit vor, eine Aufenthaltsgenehmigung zu erteilen, um die Paarbeziehung zu festigen und sich zu prüfen, ob ernsthaft an eine gemeinsame Zukunft zu denken ist. Daher sind viele Paare gezwungen, zu einem Zeitpunkt zu heiraten, der ihnen durch das Gesetz diktiert wird. Denn erst der Trauschein bringt der ausländischen Partnerin oder dem ausländischen Partner die zunächst befristete Aufenthaltsgenehmigung. Den Zeitpunkt der Eheschließung nicht selbst bestimmen zu können, empfinden viele Paare als äußerst diskriminierend.

Bis das Paar jedoch heiraten kann, müssen noch etliche Schwierigkeiten überwunden werden, die wiederum durch die Betroffenen als Diskriminierung und gar als Schikane erlebt werden. Es sind Dokumente zur Eheschließung zu beschaffen, die von der deutschen Botschaft in dem jeweiligen Herkunftsland legalisiert werden müssen. Dies gestaltet sich oft schwierig: die Dokumente in vielen Ländern außerhalb der EU entsprechen nicht den deutschen Standards.

Während die Zweck-, Versorgungs- und Vernunftsehen gesellschaftlich toleriert werden, stehen die binationalen Ehen unter einer strengen Überwachung der Ausländerbehörde und der Polizei, hinter jeder binationalen Ehe könnte doch eine Scheinehe stecken.

Rechtlich unterstehen binationale Ehen mit auBereuropäischer Beteiligung dem Aufenthaltsgesetz. Die ausländische Partnerin oder der ausländische Partner genießt beschränktes Bürgerrecht ungeach-

\section{Zümrüt} Turan-Schnieders

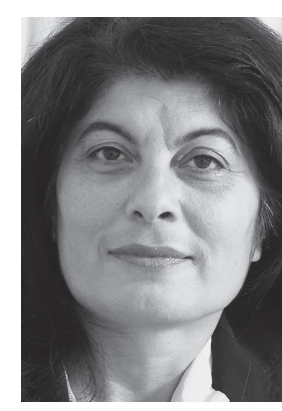

Mitglied der Kommission Zivil-, Familienund Erbrecht, Recht anderer Lebensgemeinschaften des djb, Rechtsanwältin, Hanau 


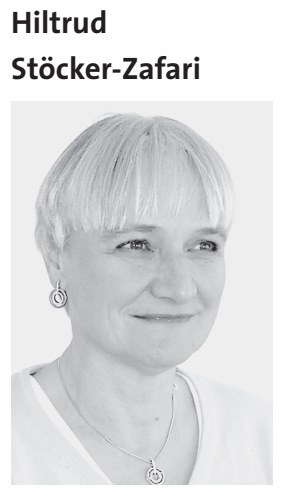

Leiterin des Fachbereichs interkulturelle Arbeit in der Bundesgeschäftsstelle des Verbands binationaler Familien und Partnerschaften, iaf e.V. tet dessen, dass ihr oder sein Aufenthaltsrecht in den ersten zwei Jahren an die eheliche Lebensgemeinschaft gebunden ist, darf sie oder er nicht wählen und findet wegen fehlender Sprachkenntnisse oder vor allem einer nicht anerkannten Ausbildung oft keinen Arbeitsplatz.

Am 28. August 2007 traten Änderungen im Aufenthaltsgesetz in Kraft, die den Ehegattennachzug aus Ländern außerhalb der Europäischen Union restriktiver als zuvor fassen. Nunmehr sollen die Ehegatten von Deutschen (ebenso wie von Ausländern) ein Mindestalter von 18 Jahren erreicht haben und vor allem Kenntnisse der deutschen Sprache auf dem Niveau A1 des europäischen Referenzrahmens nachweisen ( $\mathbb{S} 30$ AufenthG). Niveau 1 wird als die niedrigste Ebene einer generativen Sprache angesehen. Lernende können vertraute, alltägliche Ausdrücke und ganz einfache Sätze verstehen und verwenden, die auf die Befriedigung konkreter Bedürfnisse zielen; können sich und andere vorstellen und anderen Leuten Fragen zu ihrer Person stellen - z.B. wo sie wohnen, was für Leute sie kennen oder was für Dinge sie haben - und können auf Fragen dieser Art Antwort geben, sich auf einfache Art verständigen, wenn die Gesprächspartnerinnen oder Gesprächspartner langsam und deutlich sprechen und bereit sind zu helfen.

Diese Änderungen wurden mit der Begründung eingeführt, dass dadurch die Integration in Deutschland gefördert und Zwangsheiraten verhindert werden.

Die neue Regelung hat eindeutig negative Auswirkungen auf binationale Ehen (sowie auf eingewanderte Familien), ohne dabei geeignet zu sein, die Integration zu fördern oder Zwangsehen $\mathrm{zu}$ verhindern. Vielmehr wird die grundrechtlich gesicherte Position des Paares und der Familie verletzt (Art. 6 GG und Art. 8 Europäische Menschenrechtskonvention).

Das Spracherfordernis steht in keinem sachlichen Zusammenhang mit seinem vorgeblichen Ziel, Scheinehen zu verhindern. Die Neuregelung ist ungeeignet, um Zwangsheiraten zu verhindern. Zwangsheiraten sind in Zusammenhang mit patriarchalischen Strukturen zu sehen, sich aus ihnen befreien zu können, setzt vor allem Kenntnis nicht nur über Infrastruktur voraus.

Allein die Forderung, sich in einfacher Art in Deutsch verständigen zu können, stellt eine Hürde für die Paare dar.

Von vielen Paaren wird die Neuregelung, Deutschkenntnisse vor der Einreise zu erbringen, als ein Instrument angesehen, mit dem der Ehegattennachzug unterbunden werden soll.
Die Mitarbeiterinnen und Mitarbeiter in den deutschen Auslandsvertretungen setzen sogar in vielen Fällen für die Antragstellung die Vorlage des Zertifikats des deutschen Goethe-Instituts voraus. Obwohl das Auswärtige Amt die Auslandsvertretungen ausdrücklich - wenn auch nachträglich - darauf hingewiesen hat, dass Anträge auch ohne das Zertifikat des Goethe-Instituts entgegenzunehmen sind, wird dies oft nicht befolgt. Damit wird den Antragstellerinnen und Antragstellern der Rechtsweg beschnitten.

Der Besuch von Sprachschulen im Herkunftsland verursacht zusätzliche Kosten, die für viele Menschen eine extreme Belastung darstellen. Ratsuchende aus aller Welt berichten, dass die Kurse beim Goethe-Institut erforderlich sind, um überhaupt eine Chance zu haben, die Prüfung bestehen zu können. Die Kurse sind entsprechend teuer, oft übersteigen sie ein durchschnittliches Monatsgehalt in den Herkunftsländern. Hinzu kommt, dass die Kurse überwiegend in den Hauptstädten angeboten werden. Viele Ehegatten sind somit gezwungen, lange Wege auf sich zu nehmen bzw. bei Verwandten unterzukommen, die oft sehr beengt wohnen, oder sich in Hotels und Pensionen einzumieten. Diese Kosten können allein von den Ehegatten in den Drittstaaten nicht aufgebracht werden. Sie sind damit abhängig von der Zahlung der Partnerin oder des Partners im Bundesgebiet. Doch auch diese haben in der Regel nur begrenzte Mittel zur Verfügung. Die Vorgabe, Deutsch im Herkunftsland nicht nur unter finanziell schwierigen Bedingungen zu lernen, lässt den Schluss zu, dass der Gesetzgeber offensichtlich nur finanzstarke Familien als Zuwanderer wünscht.

Besonders hart sind Paare getroffen, wenn der Ehegatte wenig bzw. gar nicht alphabetisiert ist. Ihnen wird ein eheliches Leben im Bundesgebiet auf Dauer verwehrt, wenn an dem gesetzlichen Spracherfordernis festgehalten wird.

Die Verfassungsmäßigkeit dieser Sprachanforderungen, vor allem im Hinblick auf die Grundsatzentscheidung des Bundesverfassungsgerichts vom 12. Mai $1987^{1}$, ist sehr zweifelhaft. In dieser Grundsatzentscheidung zu möglichen Beschränkungen des Familiennachzugs durch Einführung eines Nachzugsalters und/oder Wartezeiten hat der 2. Senat unzweifelhaft die Verfassungswidrigkeit einer Wartezeitregelung von drei Jahren betont.

Paare fühlen sich in ihrer Partnerwahl diskriminiert, weil das Spracherfordernis auch gegen das verfassungsrechtliche Willkürverbot verstößt. Denn es kommt für das Verlangen nach den

1 BVerfG $76,1 \mathrm{ff}$. 
Sprachkenntnissen nicht auf die Staatsangehörigkeit des Nachziehenden, sondern desjenigen an, zu dem der Nachzug erfolgt. Die Konsequenz von $\mathbb{S} 30$ Abs. 1 Satz 3 Nr. 4 AufenthG i.V.m. $\mathbb{S} \mathbb{S} 16,41$ AufenthV lässt sich an folgenden Beispielen verdeutlichen:

- Eine Amerikanerin, die einen Amerikaner heiratet, muss für den Familiennachzug keine Kenntnisse der deutschen Sprache nachweisen.

- Eine Amerikanerin, die einen Türken heiratet, muss für den Familiennachzug Kenntnisse der deutschen Sprache nachweisen.

- Eine Amerikanerin, die einen Italiener heiratet, muss für den Familiennachzug keine Kenntnisse der deutschen Sprache nachweisen.

- Eine Amerikanerin, die einen Deutschen heiratet, muss für den Familiennachzug Kenntnisse der deutschen Sprache nachweisen, ausgenommen es sind deutsche Kinder aus der Ehe hervorgegangen oder der deutsche Ehemann war oder ist im europäischen Ausland grenzüberschreitend erwerbstätig.

Es gibt keine wissenschaftlichen Erkenntnisse, wonach die Ehegatten der Staatsangehörigen aus den begünstigten Ländern besonders leicht oder besonders schnell die deutsche Sprache erlernen würden. Sprachbegabung hängt sicher nicht von der Staatsangehörigkeit ab. Weiter wird bei Einreise auf Sprachkenntnisse verzichtet, wenn „ein erkennbar geringer Integrationsbedarf besteht" oder die Ehe schon bei Einreise der Ausländerin oder des Ausländers bestand, zu der oder dem der Nachzug erfolgt, oder der Ehegatte auf Grund einer Behinderung einfache Sprachkenntnisse nicht nachweisen kann.

Daneben ist zu beachten, dass für Kinder die Anwesenheit beider Elternteile in der frühesten Kindheit und der Phase unmittelbar nach der Geburt von besonderer Bedeutung ist, wie vom Bundesverfassungsgericht in ständiger Rechtsprechung ${ }^{2}$ zugrunde gelegt.

Die Voraussetzung des Spracherfordernis ist auch nicht mit den Regelungen der Familiennachzugsrichtlinie vereinbar, da die Richtlinie nur bestimmt, den Nachzug von der Teilnahme an Integrationsmaßnahmen abhängig zu machen, ohne dass hiermit der Erwerb eines bestimmten Sprachniveaus zu einer Nachzugsbedingung gemacht werden darf. Zum anderen können Familienangehörige nur auf Integrationsmaßnahmen in ihrem Heimatland verwiesen werden, wenn entsprechende Maßnahmen überhaupt angeboten und erreichbar sind.

Die für den Ehegattennachzug zu einer oder einem Deutschen einschlägige Norm setzt nicht voraus, dass der Ehegattennachzug ausnahmsweise davon abhängig gemacht werden kann, dass der Lebensunterhalt beider Ehepartner durch eigenes Einkommen oder sonstige eigene Mittel gesichert ist. Doch wird in Ausnahmefällen der Nachzug zur deutschen Ehegattin oder zum deutschen Ehegatten von der Sicherung des Lebensunterhalts abhängig gemacht. Nur die Gesetzesbe- gründung gibt den entscheidenden Hinweis auf die gewollte Differenzierung:

„Durch den neu eingefügten Satz 3 kann der Ehegattennachzug zu Deutschen bei Vorliegen besonderer Umstände von der Sicherung des Lebensunterhalts abhängig gemacht werden. Besondere Umstände liegen bei Personen vor, denen die Begründung der ehelichen Lebensgemeinschaft im Ausland zumutbar ist. Dies kommt insbesondere bei Doppelstaatlern in Bezug auf das Land in Betracht, dessen Staatsangehörigkeit sie neben der deutschen besitzen, oder bei Deutschen, die geraume Zeit im Herkunftsland des Ehegatten gelebt und gearbeitet haben und die Sprache dieses Staates sprechen.

Bei Ausländern, die mit der Perspektive eines dauerhaften Aufenthalts nach Deutschland zuwandern, findet in einem nicht unerheblichen Maße ein direkter Zuzug in die sozialen Sicherungssysteme statt. Beleg hierfür ist der Anteil von neu zugewanderten Ausländern, die von der Zahlung eines Teilnahmebeitrages bei Besuch eines Integrationskurses befreit wurden. Eine Befreiung von der Zahlung des Kostenbeitrages erfolgt nach $\mathbb{S}$ Abs. 2 der Integrationskursverordnung nur, wenn der Ausländer Leistungen nach dem Zweiten Buch Sozialgesetzbuch oder Hilfe zum Lebensunterhalt nach dem Zwölften Buch Sozialgesetzbuch bezieht. Im Jahr 2005 hatte das Bundesamt für Migration und Flüchtlinge 28,4 Prozent der neu zugewanderten Ausländer, die nach $\mathbb{4} 44$ Abs. 1 einen Anspruch auf Teilnahme am Integrationskurs haben, von der Zahlung des Kostenbeitrages aufgrund nachgewiesenen Bezugs staatlicher Sozialleistungen befreit. Im Jahr 2006 waren bis zum 30. September 2006 von 43.334 neu zugewanderten Ausländern 11.376 von den Kosten befreit. Dies entspricht einer Quote von 26,3 Prozent.

Die Neuregelung ist zugleich aus integrationspolitischen Gründen geboten. Die Pflicht zum Nachweis der Lebensunterhaltssicherung bietet für Ausländer, die die deutsche Staatsangehörigkeit erworben haben und ihren Ehepartner nachziehen lassen bzw. die sich diese Möglichkeit offen halten wollen, einen Anreiz zur Integration. Die bisherige Privilegierung des Ehegattennachzugs zu Deutschen ermöglichte es zudem, allein durch Vortäuschen einer ehelichen Lebensgemeinschaft einen Aufenthaltstitel zu verschaffen. Die Neuregelung dient somit auch dazu, die Missbrauchsmöglichkeiten einzuschränken."3

Die Begründung macht deutlich, dass hier eine Differenzierung nach dem Kriterium „,biologischer Deutscher - eingebürgerter Deutscher" und damit eine rassistische Diskriminierung aufgrund der Herkunft vorgenommen wird.

Gewiss ist, dass das Bundesverfassungsgericht zu prüfen haben wird, ob deutschen Staatsangehörigen zur (Wieder-) Herstellung der Ehe und der Familie eine „Folgepflicht“ zur Ehegattin oder zum Ehegatten im Ausland auferlegt werden darf.

2 Zuletzt: BVerfG, B. v. 9.1.2009 - 2 BvR 1064/o8.

3 Gesetzesbegründung, BT-Drs. 16/5065, S. 171. 